INPLASY

PROTOCOL

To cite: Long et al. Surgical strategies of synchronous colorectal liver metastases: a meta-analysis of RCT and case-matched retrospective studies. Inplasy protocol 202180076. doi: 10.37766/inplasy2021.8.0076

Received: 20 August 2021

Published: 20 August 2021

Corresponding author: Guojie Long

785964542@qq.com

Author Affiliation:

The Sixth Affiliated Hospital, Sun Yat-sen University.

Support: None.

Review Stage at time of this submission: Piloting of the study selection process.

Conflicts of interest:

None declared.

\section{Surgical strategies of synchronous colorectal liver metastases: a meta- analysis of RCT and case-matched retrospective studies}

Long, GJ1; Pan, WD².

Review question / Objective: The ideal treatment approach for colorectal cancer with synchronous liver metastases remains debated. We performed a meta-analysis comparing the bowel-first strategy, simultaneous strategy, and the liver-first strategy. Although there are many similar systematic reviews, the heterogeneity is high because the studies included were retrospective. This systematic review reduces heterogeneity by including randomized controlled trials and retrospective case-matching studies, which to provide a higher level of evidence on the issue. Also, to identify risk factors that affect survival, we also performe a meta-analysis of the results of multivariate analyses of survival in the included studies.

Condition being studied: Synchronous colorectal liver metastases, Hepatectomy

INPLASY registration number: This protocol was registered with the International Platform of Registered Systematic Review and Meta-Analysis Protocols (INPLASY) on 20 August 2021 and was last updated on 20 August 2021 (registration number INPLASY202180076).

\section{INTRODUCTION}

Review question / Objective: The ideal treatment approach for colorectal cancer with synchronous liver metastases remains debated. We performed a meta-analysis comparing the bowel-first strategy, simultaneous strategy, and the liver-first strategy. Although there are many similar systematic reviews, the heterogeneity is high because the studies included were retrospective. This systematic review reduces heterogeneity by including randomized controlled trials and retrospective case-matching studies, which to provide a higher level of evidence on the issue. Also, to identify risk factors that affect survival, we also performe a 
meta-analysis of the results of multivariate analyses of survival in the included studies.

Condition being studied: Synchronous colorectal liver metastases, Hepatectomy.

\section{METHODS}

Search strategy: A systemic search of online database including PubMed, Web of Science, Cochrane Library, and Embase was performed until August 11, 2021. No language restriction. The terms include "Synchronous colorectal liver metastases", "simultanous" and "delayed". We can provide more retrieval details if required.

Participant or population: Inclusion: Patients with synchronous colorectal liver metastases undergoing surgical treatment(simultaneous, bowel-first or liver-first). Exclusion: None.

Intervention: Simultaneous strategy: This means a single operation (resection or ablation, etc.) to treat both the primary tumor and the metastatic tumor.

Comparator: Delayed strategy: This means staging treatment of primary and secondary tumors, including bowel-first and liver first.

Study designs to be included: Randomized controlled trials and retrospective casematching studies.

Eligibility criteria: Inclusion: Patients with synchronous colorectal liver metastases undergoing surgical treatment (simultaneous, bowel-first or liver-first).

Information sources: Electronic databases, contact with authors, trial registers, or grey literature.

Main outcome(s): 3- and 5-year overall survival rate, 3- and 5-year disease free survival rate, postopertive complication, risk factors of survival.

Additional outcome(s): Operative time, blood loss, transfusion requirements, length of hospital stay, and treatment completion rate.

Data management: All the important information was recorded in a Microsoft excel database, such as baseline details, postoperative complications, intraoperative complications (blood loss and operating time), and long-term outcomes (mortality, 3- and 5-year overall survival rate, 3- and 5year disease free survival rate, and length of hospital stay and postoperative stay). Data extraction was performed independently by two researchers. The extracted data was double-checked by a third researcher. Disagreements were discussed with senior researcher until consensus was reached.

Quality assessment / Risk of bias analysis: The Cochrane risk of bias was used to assess quality of the RCT and the Newcastle-Ottawa scale (NOS) were used to evaluate the quality of observational studies.

Strategy of data synthesis: A meta-analysis will be used for pooling the data if the heterogeneity levels are appropriate. For dichotomous outcomes and continuous outcomes, we used the odds ratio (OR) with $95 \%$ confidence intervals $(95 \% \mathrm{Cls})$ and weight mean difference (WMD) or standardized mean difference (SMD) with $95 \%$ Cls to evaluate, respectively.

Subgroup analysis: A subgroup analysis will be conducted according to study type.

Sensitivity analysis: Sensitivity analysis is conducted by removing each study in the analysis. If the final conclusion of each item remains unchanged and the effect value changes little, it indicates that the analysis results are credible.

Language: No language limits.

Country(ies) involved: China.

Other relevant information: This is the first meta-analysis to include randomized controlled and case-matched retrospective studies on this topic, and the first meta- 
analysis to review risk factors affecting survival.

Keywords: Colorectal cancer, Synchronous liver metastases, Bowel-first, liver-first, simultaneous strategy, Meta-analysis.

Contributions of each author:

Author 1 - Guojie Long - The author searched the database, analyzed the data and drafted the manuscript.

Email: 785964542@qq.com

Author 2 - Weidong Pan - The author guided the whole review.

Email: 1710688166@qq.com 